\title{
Addressing Color Blind Awareness in the Classroom
}

\author{
Kevin Berisso* \\ Department of Engineering Technology, University of Memphis, Memphis USA \\ *Corresponding author: kberisso@memphis.edu
}

\begin{abstract}
On average, one out of every twelve men and one out of every 200 women do not see colors the same as everyone else. And yet engineering technology programs, not to mention industry, to often fail to take these people into account. This article discusses the types of color vision deficiencies (which are often grouped together under the misnomer of color blindness) that exist and some of the tools that will allow those who can see the full color spectrum to view things as if they had a color vision deficiency. Targeted towards educators as a call-to-action, and not intended as an article on how to teach color vision deficiencies to students, the goal of this article is to educate professors so that they can better get their messages across to those students who suffer from color vision deficiencies. Finally, a number of suggestions are offered on how to choose colors such that they do not cause confusion for those that suffer from color vision deficiencies.
\end{abstract}

Keywords: color blindness, color vision deficiencies, color tools

Cite This Article: Kevin Berisso, "Addressing Color Blind Awareness in the Classroom." Journal of Business and Management Sciences, vol. 6, no. 3 (2018): 93-99. doi: 10.12691/jbms-6-3-5.

\section{Introduction}

For approximately one in twelve men, or one in 200 women [1], the MARV logo at the beginning of the 2014 movie "Kingsman: The Secret Service" (see Figure 1) is an indecipherable collection of random dots that have absolutely no meaning as these people suffer from one form of color vision deficiency (CVD) or another, which is commonly misnamed as color blindness. For the remaining eleven men, or 199 women, the logo was overwhelmingly obvious. Meant as a joking acknowledgement to MARV's owner who suffers from CVD [2] the logo nonetheless raises the issue of how colors are selected for general consumption.

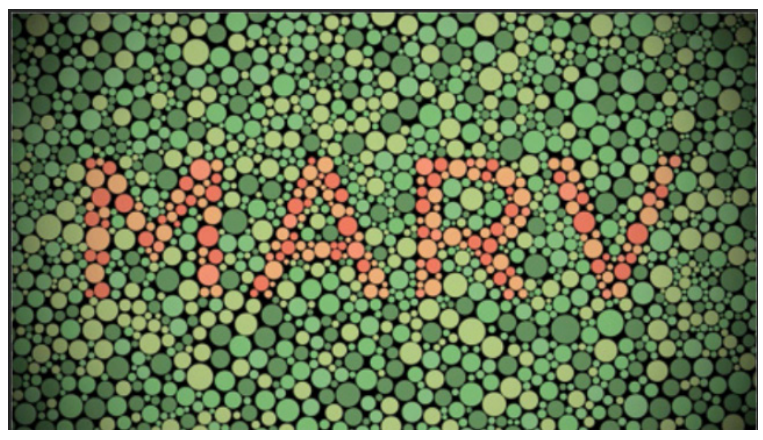

Figure 1. (Color online) MARV logo from the 2014 movie "Kingsman: The Secret Service" with the name MARV embedded in the middle of the screen. An example of the logo can be found on YouTube at www.youtube.com/watch?v=UPm1ZFwE4zQ

It should be noted at this point that most CVD people are not truly "color blind" despite the common misuse of the term. In fact, the author is functionally red-green color blind but can still see both red and green, just not as well or in the same way as everyone else. A more accurate description of the condition would be to call the author CVD. During their career, the author has taught visually based computer programming classes (using Microsoft's Visual Studio), computer application classes (e.g. MS Word, MS PowerPoint, MS Excel) and automation classes that included computerized human machine interfaces. As a standard part of these classes, a brief mention of selecting colors with an eye towards ensuring significant contrast was included, but a comprehensive discussion of CVD was not traditionally undertaken. After seeing the MARV logo and being involved in the redesign of the University's webpages, it was realized that educators needed to be better informed as to the issues and limitations surrounding those who have some level of color vision deficiency.

As educators, the goal of any presentation or hand out is to impart the information in such a way as to allow the maximum number of students to understand and learn the material. However, if the educator doesn't suffer from a CVD there exists the potential for developing materials that are difficult to understand or interpret by those that do have CVDs. And this issue crosses all of the Engineering Technology (ET) disciplines, not just those in the graphics arts. In manufacturing classes signs, work instructions and process animations often rely on color coding. Quality assurance software could use color coding to indicate out of control processes. Electronics classes use color coding on resistors to indicate both resistance values and tolerances. Electrical wiring harnesses use wire colors to designate usage. A majority of the electrical devices in use, especially those that are used for trouble shooting, use multi-colored LEDs to indicate states. And graphics software (e.g. AutoCad, Solid Edge, Photoshop, Illustrator, etc.) are heavily color based in terms of both the generated output as well as the navigation within the software (e.g. 
color coded layers, masks, etc.). Even those teaching in more business oriented areas of ET need to be cognizant of the impacts of CVDs as much of the graphing and charting software uses color to distinguish between data sets or between positive and negative values.

This paper will provide a brief overview on what CVD is, address the primary misconception about CVDs, how it impacts the portion of the population that suffers from it, and what can be done to help ensure that those with CVDs are not put at a disadvantage. Tools that allow the reader to preview what a CVD person experiences will be discussed as well as some pointers on how to select colors that will expand, not limit, the view-ability of whatever is being worked on. By the end of this article, the reader will be able to not only better understand how to avoid some of the pitfalls that cause CVD people issues, but will be to develop better class materials; ones that everyone can read and understand.

\section{Background - About Color Vision Deficiencies}

The human eye sees color via a collection of cones at the back of the eye. Numbering in the 6 to 7 million range, the cones can be divided into red sensing (64\%), green sensing (32\%) and blue sensing (2\%) [3]. It is the combination of the responses from each of these cones that results in the ability to see color (referred to as trichromatic vision). It is when one or more of these sets of cones break down that problems occur. There are three types of color vision deficiencies; anomalous trichromacy, dichromacy and monochromacy. In general, there are two ways for someone to acquire a color vision deficiency; genetics (they are born with it) or they suffer from some sort of accident or illness. In either case, a discussion of the biology and mechanics of the human eye are well beyond the scope of this article. Instead, a review of the types of color blindness will be discussed without regard as to how the person may have gotten to that point.

As previously mentioned, someone who has a fully functioning set of cones is considered to have trichromatic vision. If any or all of the cones (red, green or blue) have a shifted sensitivity, the person is considered an anomalous trichromate since they are able to "see" all three colors - just not correctly. However, if one set of cones are missing or are not responding at all, the person is considered a dichromate. For these people, the ability to process the missing color is not possible, resulting in an ability to see colors, but not all colors. Finally, the third possibility is for a person to be monochromatic. In this case, they are missing two or more cone types resulting in the inability to see any color beyond what if often referred to as grayscale. Table 1, lists the approximate percentages in which the various types of color deficiencies naturally occur for both men and women. Since the color deficiency gene is recessive [5], the lower occurrence rates in women is expected.

There are numerous tests available to aid in the determination of whether a person has a color deficiency or not. The one that most people are aware of is the Ishihara test which uses a series of colored dots on a pseudoisochromatic plate in which numbers or lines have been embedded (see Figure 2). There are a few different versions of the Ishihara test that incorporate differing numbers of plates, normally ranging from 18 to 36 . Able to identify red and green deficiencies, the Ishihara test is not able to identify blue/yellow issues with the plates that were originally developed. These plates are grouped into one of five categories; legible to all, read differently by normal and anomalous vision, legible only to normal vision, legible only to anomalous vision, and read differently by deutranopes and protanopes [6]. While most of the plates have Arabic numbers, some have lines and there is a set of shapes designed for children or illiterate subjects. A person is tested by being exposed to each plate in turn and their answers, when combined, indicated if they had normal color vision or a color deficiency and if so, whether it the deficiency is protan (red) or deutan (green) based.

Other color deficiency test tools include the use of an anomaloscope, colored tiles/discs that need to be put in sequence and lanterns (or colored light sets). The anomaloscope is a device that uses yellow light and a combination of red and green to generate yellow. The person being tested will try and combine the red and green levels to match the yellow that they see. Through the use of the anomaloscope, a person's exact type and level of color deficiency can be accurately identified [7]. Lanterns were introduced by the maritime industry to test employees on the lights that were used on ships [6].

Table 1. Breakdown of naturally occurring color deficiency types for men (M) and women (W) [4]

\begin{tabular}{|c|c|c|c|c|}
\hline \multirow{2}{*}{ Name } & \multirow{2}{*}{ Issue } & \multirow{2}{*}{ Functional blindness } & \multicolumn{2}{|c|}{$\%$ afflicted } \\
\hline & & & M & W \\
\hline \multicolumn{5}{|c|}{ Anomalous trichromatic } \\
\hline Tritanomaly & Malfunctioning blue cone & Blue-Yellow & \multicolumn{2}{|c|}{$0.0002 \%$} \\
\hline Deuteranomaly & Malfunctioning green cone & Red-Green & $4.63 \%$ & $0.36 \%$ \\
\hline Protanomaly & Malfunctioning red cone & Red-Green & $1.08 \%$ & $0.03 \%$ \\
\hline \multicolumn{5}{|l|}{ Dichromatic } \\
\hline Tritanopia & Missing blue cone & Blue-Yellow & \multicolumn{2}{|c|}{$0.0002 \%$} \\
\hline Deuteranopia & Missing green cone & Red-Green & $1.27 \%$ & $0.01 \%$ \\
\hline Protanopia & Missing red cone & Red-Green & $1.01 \%$ & $0.02 \%$ \\
\hline Monochromatic & Missing two or more cones & Fully color blind & \multicolumn{2}{|c|}{$0.00003 \%$} \\
\hline
\end{tabular}




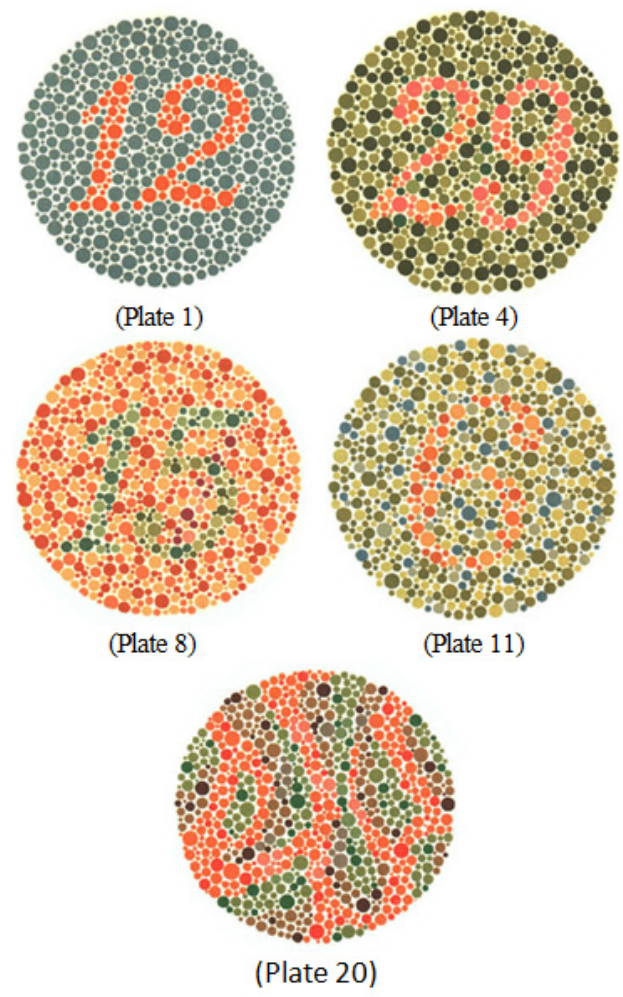

Figure 2. (Color online) Sample Ishihara plates. Everyone should be able to see the " 12 " in plate 1 . Plate 4 is " 29 " but CVDs may see "70". Plate 8 is " 15 " but CVDs may see " 17 ". Plate 11 is " 6 " but many CDVs will not see anything. Plate 20 has nothing in it but CDVs may see " 45 ". (www.color-blindness.com/color-blindness-tests/)

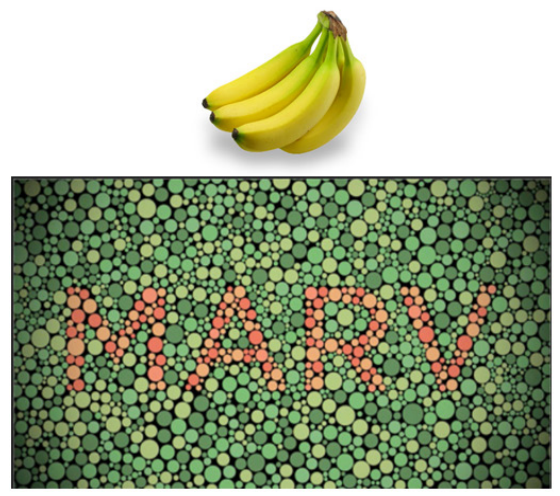

The results for those that have either anomalous trichromatic or dichromatic vision is that everyday activities can be a challenge. As shown in Figure 3, the left images are uncorrected images whereas the right images have had a filter applied so that those with full color sensitivity can experience what those with red or green based deficiencies see. As can be seen with the bananas, the green weak/missing CVD person has no way of telling if they are ripe or not since both the left and the right images look the same - yellow. Likewise with the MARV logo; the CVD person does not see the logo, but just a collection of dots.

Software is another area that can cause problems for CVD people. In the images shown in Figure 4, the round color indicators for "Tags locked", "Reject tag set" and "Tag lock failed" look the same to the author (who actually wrote the software). A slightly better choice for the "Tags locked" circle would have been the far right image, as the more common anomalous trichromatics would be able to see that the circle was green, even if deuteranopia sufferers would not be able to see the green.

Online test engines and tools exist which can help the reader to determine if they suffer from red-green CVD. And while some of the engines do a reasonable job of testing, differences in test environments, monitor performance and potential color shifts in the capturing of the Ishihara plates may skew the results. As a result, all of the tests listed should be used with caution and if the use suspects that they may suffer from a form of CVD, they should see a qualified eye specialist.

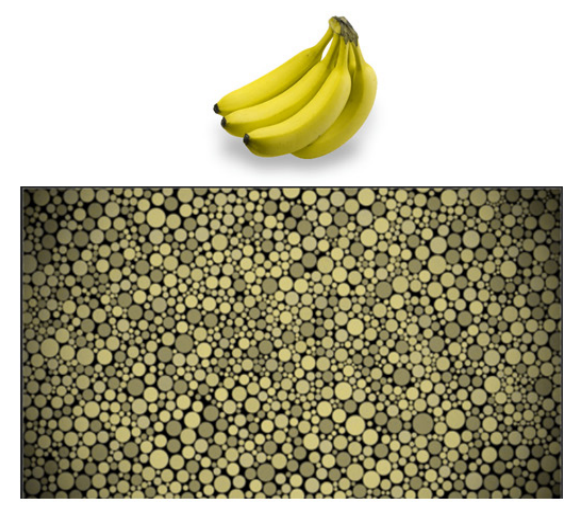

Figure 3. (Color online) What trichromatic (non-colorblind people) see (top two images) and what someone who suffers from deuteranopia sees (bottom two images) as previewed in Photoshop. Images of the bananas were retrieved from www.colourblindawareness.org/colour-blindness/livingwith-colour-vision-deficiency on 6/23/2015
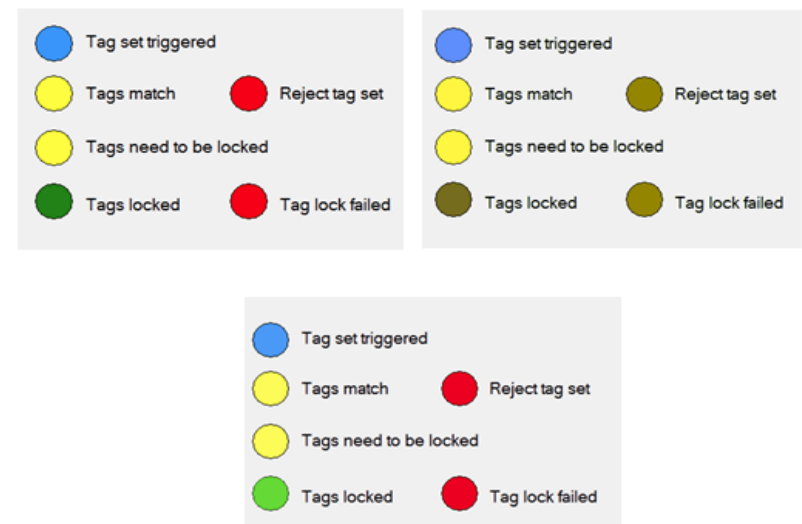

Figure 4. An example of what non-colorblind people see (top left) in a piece of software that the author developed, what people with deuteranopia see (top right) and a better choice of colors (bottom) 
Table 2. Partial list of online color vision deficiency tests

\begin{tabular}{|c|c|c|}
\hline Site & Description & Comments \\
\hline www.color-blindness.com/ishihara-38-plates-cvd-test/ & Ishihara test with results & 38 plate version \\
\hline $\begin{array}{l}\text { www.colour-blindness.com/colour-blindness- } \\
\text { tests/ishihara-colour-test-plates/ }\end{array}$ & Ishihara test & 24 plate version with descriptions of what to expect \\
\hline $\begin{array}{l}\text { www.color-blindness.com/farnsworth-munsell-100-hue- } \\
\text { color-vision-test/ }\end{array}$ & $\begin{array}{l}\text { Farnsworth-Munsell } 100 \\
\text { HueColor Vision Test }\end{array}$ & \\
\hline www.color-blindness.com/color-arrangement-test/ & Color Arrangement Test & \\
\hline $\begin{array}{l}\text { www.color-blindness.com/rgb-anomaloscope-color- } \\
\text { blindness-test/ }\end{array}$ & $\begin{array}{l}\text { RGB Anomaloscope - Color } \\
\text { Blindness Test }\end{array}$ & $\begin{array}{l}\text { The author has determined that this test is not accurate, } \\
\text { however it does give a good idea of what the test is like. }\end{array}$ \\
\hline $\begin{array}{l}\text { play.google.com/store/apps/details?id=ch.colblindor.colo } \\
\text { rblindcheck }\end{array}$ & $\begin{array}{l}\text { Cell phone Color Blind } \\
\text { Check }\end{array}$ & $\begin{array}{l}\text { An Android / Apple / Windows app that can test for } \\
\text { CVDs }\end{array}$ \\
\hline $\begin{array}{l}\text { www.colour-blindness.com/colour-blindness- } \\
\text { tests/colour-arrangement-test/ }\end{array}$ & Color arrangement test & Similar to the one from Colblindor \\
\hline
\end{tabular}

\section{Addressing Color Vision Deficiencies}

When discussing CVD, it is often extremely difficult, if not impossible, for trichromatics to understand what those that suffer from CVD are experiencing. Quite possibly the single most obvious proof of this is the fact that most people group all color vision deficiencies under the title "color blind." Since, as was previously discussed, most people with CVD are in fact able to see some color, calling it color blindness is really a misnomer. In fact, various authors have suggested that it be referred to as a "color vision deficiency" or "color vision impaired" $[5,8]$.

All too often, ET professors fail to integrate a color awareness in their curriculums design. This oversight is often due to the fact that the person teaching does not suffer from CVD, and therefore doesn't think in those terms. When developing educational materials there are three things that the instructor should consider; design suggestions, simulation tools for previewing work and the tools that assist CVD students.

\subsection{Design Suggestions}

Quite often the easiest thing to do in terms of developing a CVD friendly materials is to avoid color combinations that will cause those with CVD problems. By using patterns, annotations, hue shifts or just plain black and white [5], designers can make life easier on those with CVD. Because anomalous trichromatics are the most common CVD suffers, avoid similar shades (e.g. green and brown or blue and purple) or consider adding differing textures to the colors [9].

According to the blog on one website that discusses graphic design for CVD [9], designers should:

- Make it monochrome: Using various shades of a single color instead of multiple colors is of course the most surefire way to avoid color blindness issues. Today minimalism is all the rage, isn't it?

- Use high contrast: Color blind people can still perceive contrast, as well as differences in hue, saturation and brightness. Use these to your advantage (Hint: many color blind individuals report being able to better distinguish between bright colors rather than dim ones, which tend to blur into one another)

- Use thicker lines: Some mildly color blind people are able to see a color, but only if there's a sufficient "mass" of it. If a line of color is too thin, it won't show up as the right color.

- Don't assume colors will signal emotions in and of themselves: If you're using red to signal "bad," "warning," or "watch out," consider adding another symbolic element to get the point across to color blind viewers.

- Use texture instead: in maps and infographics, try using texture in addition to color to differentiate between objects.

Reference [10] agrees listing the following issues that CVD students will encounter, implying that the elimination of these activities, or their modification to include color, would make the activity CVD friendly.

- colour blind students cannot read litmus paper accurately

- colour blind students cannot tell the colours of different chemical solutions and would have great difficulty undertaking chemical titrations in practical chemistry exams

- colour blind students are unable to identify metals by the colour of the flame produced when the metal is burnt

- colour blind students are unable to accurately read stained slides under a microscope

- colour blind students may not be able to accurately carry out dissections in biology

- colour blind students may not be able to identify species of plants or insects correctly

- colour blind students will have difficulty fully understanding coloured diagrams in textbooks, particularly in biology

- colour blind students will have difficulty with coloured wiring, use of prisms in physics etc.

In addition to the list above, the author has noted that the reading of multi-color LEDs can cause problems for students with CVDs. Reference [11] indicates that when dealing with color, designers should do the following.

- In graphs, place the legend directly in the chart

- In graphs and maps, display the type of data for each element in a tooltip so it can be viewed on a mouseover [done with the a:hover tag] so color isn't the only attribute

- Use varied icons or varied shapes in addition to color for status icons 
- When users are required to select a color, name the color in text as well as showing the color

- To sum it up: never use color alone to indicate anything!

Both [11] and [9] suggest that when developing graphics, that the following combinations will be difficult for varying segments of the CVD population; green and red, green and brown, blue and purple, green and blue, light green and yellow, blue and gray and green and gray and green and black. Malamed also indicates that blue and yellow, yellow and violet and dark blue and black, while not as problematic, may also cause some CVD people issues.

As can be seen in the preceding paragraphs, there are no simple solutions by CVD type. Instead, the suggestions provided are applicable to all CVD types.

- Avoid using multiple colors - use various shades of the same color

- Use high contrast

- Ensure that colors are "thick" - thicker lines can be easier to see

- Use texture and textual information - don't use color alone as an indicator

\subsection{CVD Simulators}

Even given the suggestions in the previous section, when it comes time to develop something, most educators will ultimately find themselves violating at least one of the suggestions for any number of reasons. As such, when teaching to engineering technology students, it is important to be able to provide a method in which all students are able to visually understand the material whether it be graphs in Excel or graphical displays or web pages. To this end there are a number of tools available that range from simple websites to Photoshop. Table 3 is a partial list of online CVD simulations. In some cases preselected images are shown from the point of view of someone with "normal" vision and then one or more of the CVD filters are applied. Other tools allow the user to upload their own images for evaluation. Of the online simulators that the author tested, the tool at www.colorblindness.com (see Figure 5) seemed to be the most versatile in that it allowed for reasonably large images, a cross-section of file formats and all of the CVD combinations.

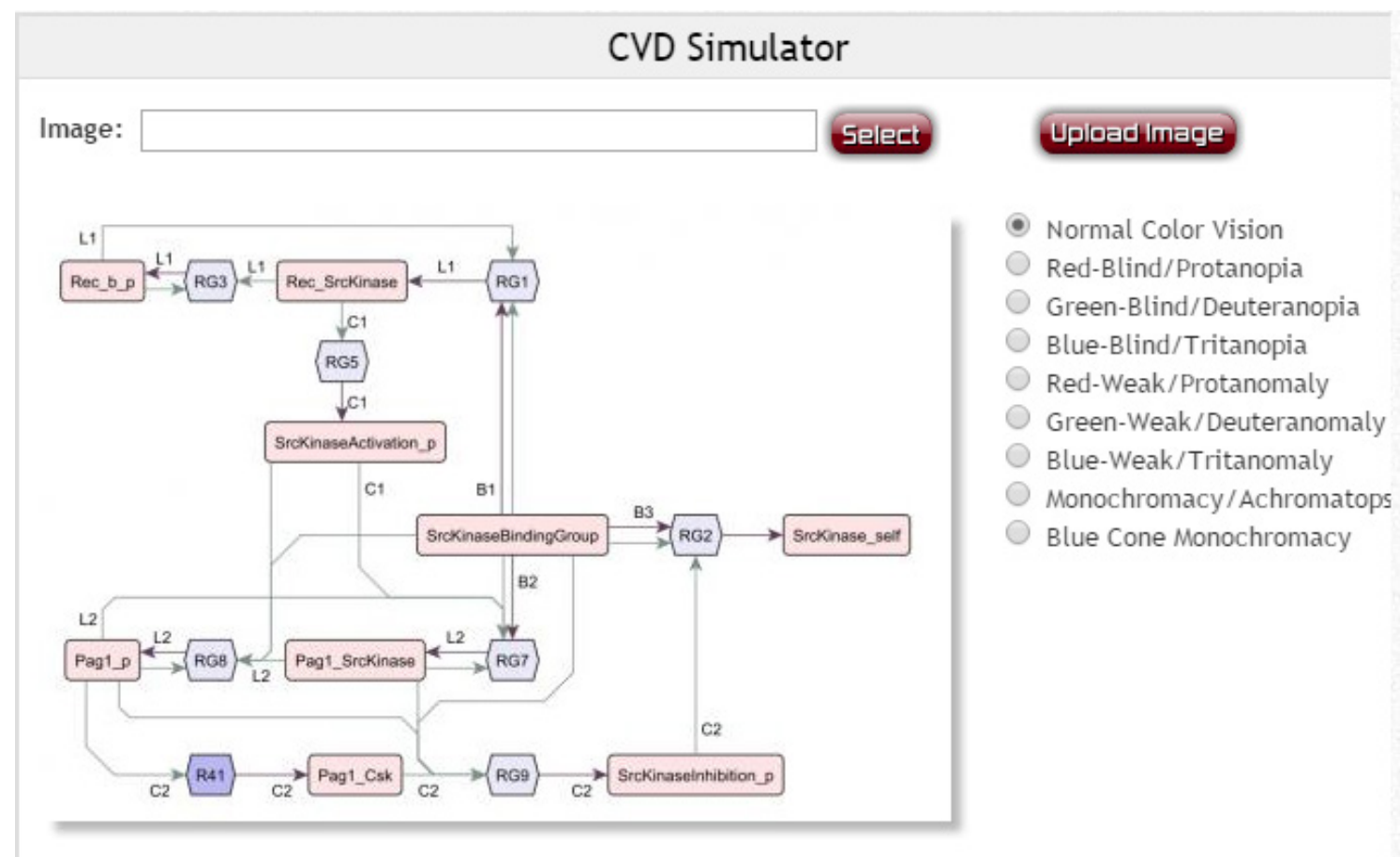

Figure 5. CVD Simulator. (www.color-blindness.com/coblis-color-blindness-simulator/)

Table 3. Listing of online CVD simulators.

\begin{tabular}{|l|l|}
\hline Site & Comments \\
\hline www.color-blindness.com/coblis-color-blindness-simulator/ & Allows user to load their own images (jpg, gif, png that are 600kb or less) \\
\hline www.colorvisiontesting.com/what\%20colorblind\%20people\%20see.htm & Examples of what "normal" colors and various CVD colors look like \\
\hline $\begin{array}{l}\text { www.colourblindawareness.org/colour-blindness/colour-blindness- } \\
\text { experience-it/ }\end{array}$ & Examples of what "normal" colors and various CVD colors look like \\
\hline www.colorfilter.wickline.org/ & $\begin{array}{l}\text { Allows the user to enter a web page and run the page through the selected } \\
\text { CVD filter. Does not seem to handle HTML5 sites very well. }\end{array}$ \\
\hline www.webexhibits.org/causesofcolor/2.html & $\begin{array}{l}\text { Examples of what "normal" colors and various CVD colors look like for } \\
\text { selected images }\end{array}$ \\
\hline colorlab.wickline.org/colorblind/colorlab/ & $\begin{array}{l}\text { Displays a color pallet that can have various CVD filters applied to show } \\
\text { what CVD people see. }\end{array}$ \\
\hline
\end{tabular}


Table 4. List of downloadable software for simulating CVD

\begin{tabular}{|l|l|}
\hline Site & Comments \\
\hline www.ryobi-sol.co.jp/visolve/en/ & Software will do a partial screen capture and can apply various CVD filters to the resulting image. \\
\hline asada.tukusi.ne.jp/cvsimulator/e/index.html & Application for use on smart phone. \\
\hline www.colororacle.org/index.html & Does not seem to do live interpretations, but instead processes the primary screen. \\
\hline
\end{tabular}

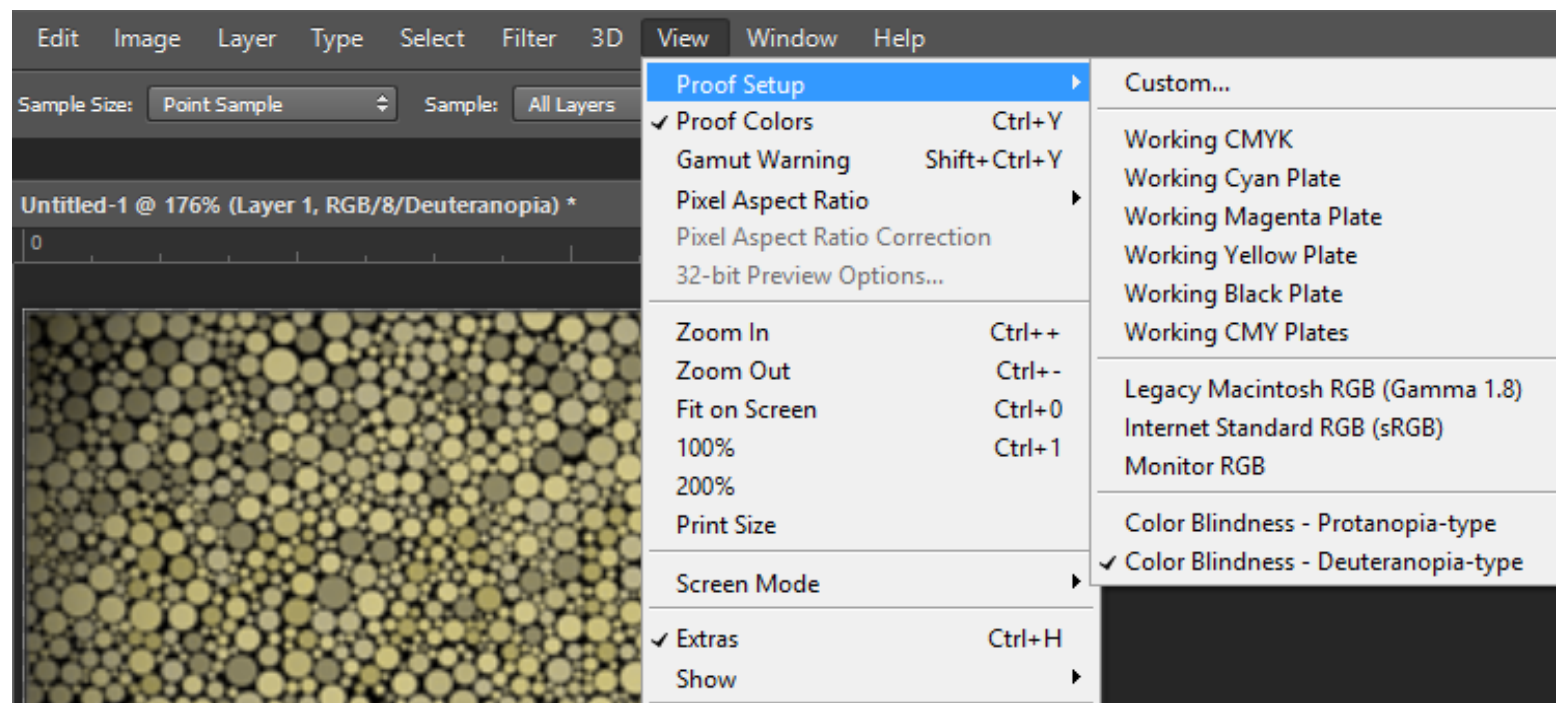

Figure 6. Photoshop CC 2014 colorblindness preview functionality

In addition to the online tools found, there are tools that can be downloaded and installed on a computer that do the same job. A few of them are listed in Table 4.

If the reader has access to Photoshop, under the View menu item is an option to proof colors (see Figure 6). This is a tool that will allow someone to view the image being worked on as if they suffered from either protanopia or deuteranopia.

\subsection{CVD Tools}

For those that have a CVD, there are tools available to help them to be able to better discern colors on the computer. Since many of these tools have limitations, it behooves the educators to be aware of the tools so that materials can be developed that are compatible with the tools available. The process by which images are adjusted to account for CVD is known as Daltonization [12]. Chrome Daltonize is a Chrome browser add-in that attempts to recolor any graphics within a web site. As can be seen in Figure 7, the Daltonization process allows someone with CVD to more easily view images that would otherwise have little to no meaning to them. Table 5, has a partial list of Chrome browser add-ins.
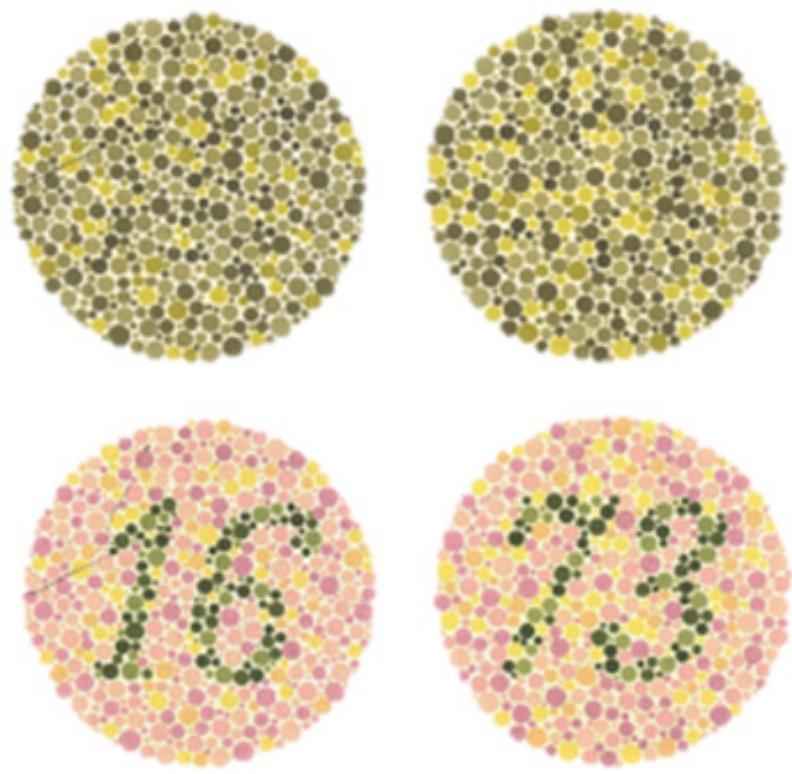

Figure 7. Upper image set is what a Protanopia would see on a website and the lower image set has been put through the "Chrome Daltonize!" add-in

Table 5. Partial list of software to help with CVD

\begin{tabular}{|l|l|}
\hline Website & App \\
\hline chrome.google.com/webstore/detail/colorblind-helper/dlonjjgpeedbkjdipiiboelenjdjjbak & Colorblind Helper \\
\hline chrome.google.com/webstore/detail/daltonaid/hdlekikjalkhdabmhjjdmloklbbmlodp & DaltonAid \\
\hline $\begin{array}{l}\text { chrome.google.com/webstore/detail/ebay-for-the-color- } \\
\text { blind/mnclemflbnmkkkcncpnakjfionmfehpa }\end{array}$ & Ebay for the Color Blind \\
\hline chrome.google.com/webstore/detail/color-enhancer/ipkjmjaledkapilfdigkgfmpekpfnkih & Color Enhancer \\
\hline
\end{tabular}




\section{Conclusions}

Color vision deficiency is not an overly debilitating affliction. And while it can be troublesome and even aggregating to those that have it, there are ways to minimize its impact. As has been shown in this article, there are a number of steps that educators can take to help ensure that their materials are CVD friendly. The use of the tools discussed make it easy to experience what someone who has CVD sees, allowing for the designer to "proof" their work. With the information presented, and the tools and filters discussed, CVD friendly materials are easily injected into just about any class.

One unexpected outcome of developing this paper was the realization that a comprehensive CVD module needs to be developed that would allow professors to present this material to students in a logical and quantifiable manner. Since a small portion of the students might suffer from CVD, care will have to be taken to ensure that they are able to actively participate in the exercises, since they wouldn't be able to tell the differences between some of the potential materials.

\section{References}

[1] “Colour vision deficiency." NHS Choices. 2014. Available: http://www.nhs.uk/conditions/Colour-visiondeficiency/Pages/Introduction.aspx. [Accessed June 17, 2015].

[2] "Marv Films - CLG Wiki." CLGWiki. Available: http://www.closinglogos.com/page/Marv+Films. [Accessed June $18,2015]$.
[3] Nave, C. R. "The Rods and Cones of the Human Eye." HyperPhysics. 2001. Available:

http://hyperphysics.phy-astr.gsu.edu/hbase/vision/rodcone.html. [Accessed on June 22, 2015].

[4] Colblindor. "Types of Color Blindness." Colblindor.com. Available: http://www.color-blindness.com/2010/03/09/types-of-colorblindness/. [Accessed June 19, 2015].

[5] Jenny, B \& Kelso, N. V. "Color Design for the Color Vision Impaired." Cartographic Perspectives, 58:61-67. Fall 2007.

[6] "Ishihara and other Colour Vision Tests." College of Optometrists. Available:

http://www.college-optometrists.org/en/college/museyeum/ online exhibitions/colour vision/ishihara.cfm. [Accessed on June 19, 2015].

[7] Williams, C. H. "Nagel's Anomaloscope for Testing Color Vision" Transactions of the American Ophthalmological Society, 14 (1): 161-165. 1915.

[8] Jefferson, L. \& Harvey, R. "An Interface to Support Color Blind Computer Users." CHI 2007 Proceedings, 2007.

[9] Bigman, A. 2015. "Why all designers need to understand color blindness." 99designs Designer Blog, February 13, 2015. Available:

http://99designs.com/designer-blog/2013/04/17/designers-need-tounderstand-color-blindness/. [Accessed on June 23, 2015].

[10] "Secondary School \& Higher Education." Colour Blind Awareness. Available: http://www.colourblindawareness.org/teachers/secondary-schoolhigher-education/. [Accessed on June 19, 2015].

[11] Malamed, C. "Designing For Color Blindness." Understanding Graphics, 2015. Available: http://understandinggraphics.com/design/designing-for-colorblindness/. [Accessed on June 23, 2015].

[12] Rasche, K., Geist, R. \& Westall, J. "Re-coloring Images for Gamuts of Lower Division.” Eurographics, 24 (3):423-432. 2005. 